\title{
TIME-RESOLVED SPECTROSCOPY OF SHORT PERIOD RS CVn SYSTEMS
}

\author{
C. Lázaro and M.J. Arévalo \\ Instituto de Astrofísica de Canarias, E-38200, La Laguna, Spain
}

\begin{abstract}
We have initiated a programme of spectroscopic observations of RS CVn short-period group, with orbital phase resolution. The systems of this group are all eclipsing binaries with both components at the Main Sequence, and most of them have similar spectral type components. The high rotational velocities and their short orbital periods (less than 1 day) hinder the spectroscopic study of these stars. We presents the first results yielded by $\mathrm{H}_{\alpha}$ line observations of the systems XY UMa and WY Cnc. Both systems were observed during 1991 with reasonably wide orbital phase coverage. The analysis of the spectra is made by comparison with a binary model, constructed from the observed spectra of normal stars of the same spectral type as the RS CVn system components. The model accounts for the partial contribution of each component at any orbital phase within eclipses.
\end{abstract}

\section{OBSERVATIONS}

The spectra presented here were obtained in 1991 during two observing runs using different telescopes. The first observations were made in February from $22 / 23$ to $24 / 25$ at the Coudé focus of the $2.2 \mathrm{~m}$. Telescope at the GermanSpanish Calar Alto Observatory (Almeria, Spain). We observed again on the nights November 23/24 to $26 / 27$ at the Coudé focus of the $1.5 \mathrm{~m}$ Spanish telescope at the same Observatory. The $2.2 \mathrm{~m}$ observations cover the spectral range $\lambda$ : $6531-6600 \AA$, with a spectral resolutions of $0.07 \AA /$ pixel. The observations made with the $1.5 \mathrm{~m}$. cover the range from $\lambda: 6460 \AA$ to $6740 \AA$, with a spectral resolutions of $0.54 \AA$ /pixel. The data reduction process has been performed using the software packet FIGARO for extraction and wavelength calibration.

One of the aims of this study is to follow the variations of the spectroscopic appearance of the RS CVn systems with orbital phase, covering the complete orbital cycle with good orbital resolution. This makes it convenient to maintain as low as possible the ratio beteween the exposure time of the individual spectra and the orbital period of the observed system. On the other hand, these systems are relatively faint: $\mathrm{V}=9.5$ for WY Cnc and V=9.8 for XY UMa (both at the maximum), and their orbital periods $0^{d} .83$ and $0^{d} .47$ for WY Cnc and XY UMa respectively. These periods preclude the possibility of long exposure times and limit the signal to noise ratio that may be achieved 
in the spectra without a severely degrading spectral and orbital resolutions. We have chosen a compromise between those opposite considerations, and thus the individual spectra spread about 0.02 in orbital phase for WY Cnc, and 0.05 in phase for XY UMa.

\section{RESULTS}

We study spectral line variations comparing the observed spectra of the RS CVn systems with a theoretical spectrum of a normal eclipsing binary. This theoretical spectrum is generated for any orbital phase from the observed spectra of two normal stars, with the same spectral type as the components of the RS CVn system. As comparison stars, we observed several bright stars with unknown active behaviour. In the model spectra presented in this work, we have used:

- HD 32147 ( $\mathrm{K5}$ V) with 70 Vir (G4 V), for the XY UMa spectra obtained at the $2.2 \mathrm{~m}$. telescope.

- HD 32147 with HR 1656 (G4 V) for the XY UMa spectra observed in at

$1.5 \mathrm{~m}$. telescope.

- HD 36395 (M2 V) with 70 Vir for the WY Cnc system.

The intrinsic brightness of each component in the binary model is taken from the MARCS models atmosphere fluxes (Gustafsson et al 1975). The limb darkening and the partial contribution of each component during the eclipse phases are properly taken into account, but non-sphericity, reflection and gravity darkening effects have been neglected at this stage. The model gives the total expected spectra plus the contribution of each component. This allows to identify easily the location of the $\mathrm{H}_{\alpha}$ emission line in the system, and to derive the ratios of line emission to photospheric fluxes for each component. XY UMa: The spectra at fases $0.24,0.0$, show $\mathrm{H}_{\alpha}$ excess with variable intensity (Figure 1 and Figure 2). Both component stars present emission at different phases.

WY Cnc: At the two orbital phases shown here, the system presents also a strong $\mathrm{H}_{\alpha}$ excess (Figure 3 and Figure 4). This emission line presents two components. The strongest can be associated to the primary component, but the pointing out the origen of the other component must be based on the complete set of data.

It seems that this behaviour has been not observed previously, as Strassmeier et al. (1988) quote strong $\mathrm{H}_{\alpha}$ absorption for the XY UMa system. Whilst giving no information on on WY Cnc. Pojmanski (1992) found $H_{\alpha}$ in XY UMa to be much weaker than in G2-G5 V stars, and strongly variable. This is indicative of the complex behaviour of these systems, adds more interest to the continuity of this kind of observations.

\section{REFERENCES}

Gustafsson,B., Bell, R.A., Eriksson,K., Nordlund, A., 1975 Astr. Ap., 42, 407. Pojmanski 1992: Private comunication

Strassmeier, K, Hall, D.S., Zeilik, M., Nelson, E., Eker, Z., and Fekel, F., 1988 Astr. Ap. Suppl., 72, 291. 

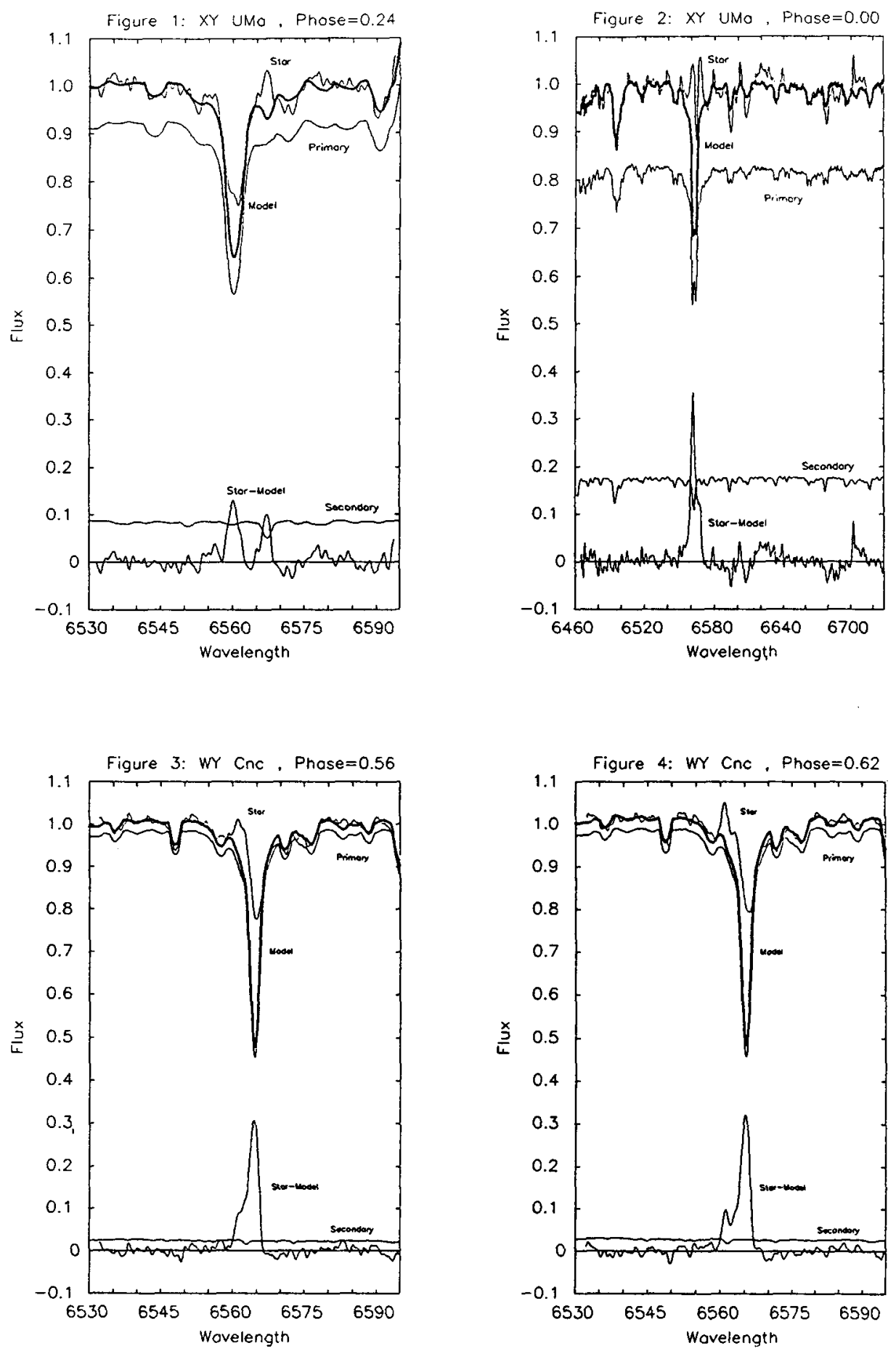